\title{
Hearing from men in Uganda: Experiences with HIV services and prevention programming, and perceptions of DREAMS-Findings from DREAMS implementation science research
}

Population Council

Follow this and additional works at: https://knowledgecommons.popcouncil.org/departments_sbsr-hiv How does access to this work benefit you? Let us know!

\section{Recommended Citation}

Population Council. 2021. "Hearing from men in Uganda: Experiences with HIV services and prevention programming, and perceptions of DREAMS-Findings from DREAMS implementation science research," DREAMS Results Brief. Washington, DC: Population Council. 


\section{HEARING FROM MEN IN UGANDA: EXPERIENCES WITH HIV SERVICES AND PREVENTION PROGRAMIMING, AND PERCEPTIONS OF DREAMS FINDINGS FROM DREAMS IMPLEMENTATION SCIENCE RESEARCH}

HIV prevention efforts across sub-Saharan Africa are increasingly focused on engaging men, for their own health and that of their partners and families. We examined experiences with HIV services and prevention programing among men in Uganda whose partners were enrolled in DREAMS ${ }^{*}$, a large-scale initiative to reduce new HIV infections among adolescent girls and young women (AGYW). ${ }^{1}$ The study is part of the Population Council's implementation science research portfolio on the DREAMS Partnership. Our studies related to male partners of AGYW were carried out in Eswatini, South Africa, and Uganda.

Engaging men/male partners is key to addressing AGYW's risk, and the DREAMS Partnership aims to do so by getting men/male partners of AGYW into HIV testing, voluntary medical male circumcision (VMMC), linkage to HIV care and antiretroviral treatment (ART), and by implementing programming to change harmful gender norms in communities, including among men/male partners.

Our previous research with male partners of AGYW in Uganda found that multiple sexual partnerships were common, and that most men had both long-term side partners as well as short-term relationships with AGYW, and that men's HIV testing decisions were made based on the stage of each relationship. ${ }^{2}$

In this brief we examine men's perceptions of DREAMS, shifts in HIV risk as DREAMS was implemented, uptake of HIV testing and VMMC services, and care experiences among men living with HIV.

*DREAMS stands for Determined, Resilient, Empowered AIDS-free, Mentored, and Safe.

\section{KEY FINDINGS}

Men were largely supportive of their partners' engagement in DREAMS. They had varying levels of involvement in DREAMS programming themselves

Men who participated in HIV prevention programming reported positive behavior change, such as improved couple communication, reduced violence, and numbers of sexual partners.

Men's-only meetings had a particular role: helping men cope together with life challenges.

Most men were routinely testing for HIV, due to convenient community-based options and DREAMS-related invitations, and positively perceived VMMC. However, additional approaches were critical to support men reluctant to test or link to care, such as messaging around the effectiveness of early antiretroviral treatment.

Men living with HIV had good engagement in HIV care, as well as high awareness viral load/viral suppression, but only moderate awareness of treatment as prevention (TasP).

Location: Peri-urban and rural settings in Gulu, Sembabule, and Mukono districts

Study Duration: 2016-2019

Funder: Bill \& Melinda Gates Foundation

Research Partners: Child Health \& Development Centre, Makerere University College of Health Sciences, School of Medicine 


\section{METHODS}

We conducted qualitative in-depth interviews with 36 male partners of AGYW enrolled in DREAMS, from May to September 2018. Our sample included 20 men (18 HIV-negative, 2 HIV-positive) who participated in an earlier round of data collection with men/male partners of AGYW and also in DREAMS activities over the course of the program. We also interviewed 16 men living with HIV who were recruited by DREAMS implementing partner organizations. Respondents were from six sites in peri-urban and rural settings within three culturally diverse districts across Uganda (see map) where DREAMS was implemented. Thematic data analyses followed a team-based, iterative process, including interpreting emerging findings with local stakeholders. Findings were quite similar across districts.

\section{MEN'S PERCEPTIONS OF DREAMS}

\section{Men were largely supportive of DREAMS.} Overall, men supported their partners' engagement in DREAMS. They particularly appreciated the program's skills-building/income generation activities and access to health information and services, which many described as benefitting their partners as well as themselves and their families.

[My partner] learned how to make soap and vaseline, there were others who were doing a number of other different things.... She also learnt how to save money, [and] she managed to do somethings at home.... When she tells me that I got
Map of study districts

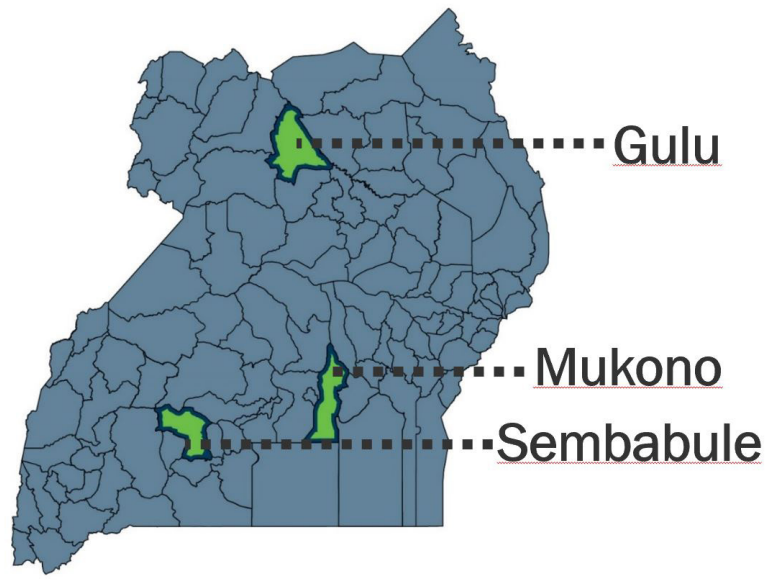

this from DREAMS, I know automatically that things have moved on well.

-Age 26, Sembabule

DREAMS has taught my wife so many things and it has changed her life positively. Now she even shares with neighbors on how to behave well in society. She also encourages people to go for HIV testing.... If I follow everything she shared with me, I will be able to live an HIV free life.

-Age 26, Mukono

\section{Men had varying levels of engagement with DREAMS.}

The DREAMS Partnership includes direct outreach to male partners of AGYW in the program in contexts like Uganda where many AGYW are married/cohabiting;

\section{Who are the $\mathbf{3 6}$ men who participated in the qualitative interviews?}

\section{Mean age 32; range 20-54}

\section{All had an AGYW partner in DREAMS (per study design)}

- Most of these partners were wives or long-term partners, but several were casual partners

- A few men had 2 partners/wives in DREAMS

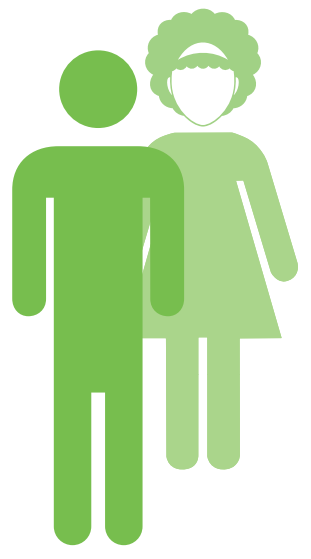

Nearly all men were employed.

Occupations included farmers, transport workers, builders/ handymen, fishermen, and other service industry workers

Half were living with HIV (per study design) 
otherwise the approach is to characterize typical male partners of AGYW and target outreach accordingly. ${ }^{1}$

Most men had engaged with DREAMS when offered HIV services by a DREAMS implementing partner (i.e., community-based organization or service provider).

Some respondents also described directly participating in educational HIV prevention programming, alongside their partners/other women (e.g., SASA!, an intensive weeks-long program focusing on critical reflection around gender norms, violence, and HIV risk ${ }^{3,4}$ ) or only among men (e.g., for “men's meetings” for partners of AGYW in DREAMS). Others consumed information second-hand from their partners in DREAMS, especially what their partner learned in the Stepping Stones program (an intensive program similar to SASA!, which in Uganda was geared mainly toward $A G Y W^{4,5}$ ), in addition to or instead of participating directly. Still others said they did not discuss DREAMSrelated programming with their partners, sometimes explaining that it was not for men.

For DREAMS, I first knew it as a program for women and therefore I have never gone there. My wives just tell me that 'we are going for education programs' and the truth is that I don't refuse them, I give them ample time to go and study because it's their time.... [And ] they come back and tell me that we have studied this and that.

-Age 39, Sembabule

It has been designed for them [i.e., young women], and I don't know what it is all about because she has never told me.

-Age 42, Sembabule

The HIV prevention programming men participated in ranged from a single 2- to 3-hour session (e.g., men's meetings) to more intensive (e.g., half-day) sessions held a few times a week for several months (e.g., SASA!).

Sessions were described as covering HIV prevention information (reducing number of sexual partners; condom use) and providing referrals to HIV services, and often also covered communication and power dynamics/violence in intimate relationships, as well as the importance of respect for others in the community and of supporting people living with HIV.

Men's meetings also were often described as intending: ...to tell us why our wives are in the DREAMS Program, the type of support services provided to empower them to become healthy and self-reliant economically.

-Age 42, Sembabule

\section{SHIFTS IN HIV RISK}

\section{Participating in HIV prevention programming supported positive behavior change.}

Men who participated in HIV prevention programming related to DREAMS commonly reported effects on improved communication and conflict resolution in relationships, and reduced violence in relationships. These changes were often described as arising out of the foundation of understanding gained from the program, about the harm of control and power in relationships and respect for others in one's community, particularly in the case of mixed gender and/or more intensive programs (e.g., SASA!).

\section{S SASA! is about how people use their power in the family.... SASA! helps us to understand that each person has power, but then how does one use his power? Participating in SASA! has helped people achieve peace in their families.}

-Age 32, Sembabule

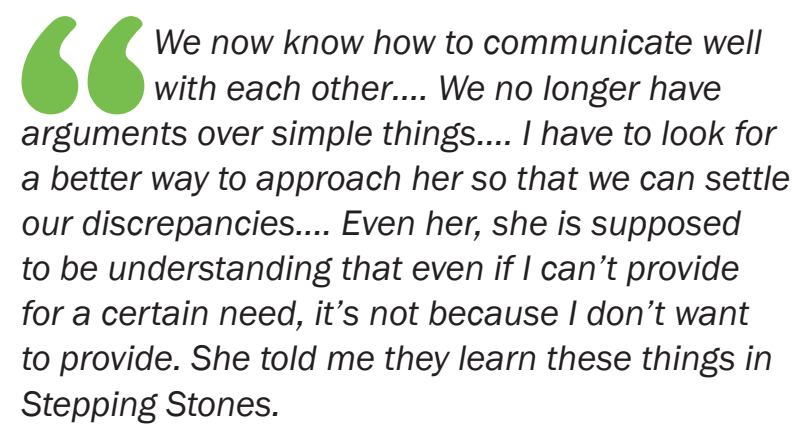

-Age 26, Mukono

Many men also reported program effects on reductions in the number of sexual partners they have. In fact, most of the men who reported multiple partners when interviewed as part of a previous study, now reported having only one partner. Partner reduction was also described as arising both out of reflecting on power dynamics in the relationship and family, as well as better understanding partner reduction as an effective way to reduce HIV risk. 
The meeting taught me, as a person, to be safe, and practice self-control in all ways of life. Having one partner and caring for our partners.... This increased my desire to protect myself against HIV infection; to stop admiring other women.

-Age 27, Sembabule

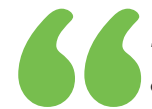

...there are men who do not know a lot about HIV and its effects so when in these meetings they are taught about HIV, that is, how it is acquired, its effects, care and treatment and its prevention.... That's when you will find one who has been having very many sexual relationships starting to reduce the number of people they sleep with or even deciding to become faithful and committed to one partner. These meetings are so insightful and they lead many to change their behavior....

-Age 38, Mukono

\section{Particular role for men's-only meetings: coping with life challenges.}

In each country, participants of men's meetings consistently described these meetings as providing opportunities for men to discuss the challenges they face and learn positive coping strategies, in addition to encouraging HIV risk reduction and linkage to services. Nearly all men who participated in men's meetings in each country did not also describe participating in other programming that included women.

\section{6 I have participated in a meeting of men in the community....we talked about everyday lives that we go through as men. The facilitator... explained the reasons why the meeting organizers invited only men to attend the meeting...they never wanted women to know men's secrets and things that affect men in relationships. We were also informed that women will be granted the opportunity to discuss the issues affecting their relationships, separately in a women's meeting.... [Our] meeting focused on the behavior of men in a relationship...[and] the challenges and benefits experienced by male partners of DREAMS girls in terms of their relationships.}

-Age 27, Sembabule

\section{HIV SERVICES-HIV TESTING AND VMMC UPTAKE}

\section{For most men, use of HIV testing had become routine-facilitated both by convenient options and DREAMS-related invitations.}

Most men described testing for HIV on a routine basis. Many attributed this to increasingly convenient and varied testing options now available in their communities. For example, along with facility-based testing (with shorter wait times and more flexible hours), there were mobile, door-to-door, and workplace testing options.

\section{....when they come, I go and test. There are times when they organize testing in our village centres...[or] in the trading centres.... Some just come without announcing, but most organizations inform the community members in advance....}

-Age 38, Mukono

Many also described being invited to test by their partner in DREAMS or a DREAMS implementing partner staff member. They appreciated receiving this personal invitation, while not always acting on it if they had already tested elsewhere recently.

Since my wife tests a lot at the organization [DREAMS], she tells me and I also get encouraged to go for testing more often when I get time. At their organization they test every 3 months.

-Age 26, Mukono

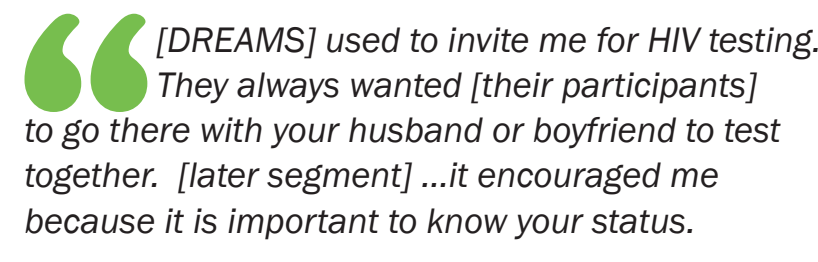

-Age 27, Mukono

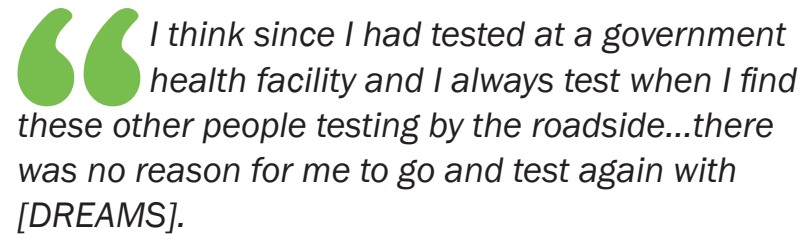

-Age 30, Mukono 
However, additional approaches were critical to support men who were reluctant to test.

HIV prevention programming like SASA! and men's meetings seemed particularly well-suited to supporting testing and linkage to HIV services for men previously hesitant to do so. For some who attended men's meetings, the recognition of and support for coping with life challenges seemed to serve as a foundation from which men felt heard, and in turn more confident taking the step to get tested.

[SASA!] was about real things in our life and most especially how we use our power in the family. [Later segment] SASA! helped me a lot. It gave me the courage to participate in HIV testing and so to know my status together with my wife...[and] increased my interest in protecting myself and my wife against HIV.

-Age 29, Sembabule

I received an invitation to attend a men's meeting...it was at that meeting that I had my first HIV test.

-Age 42, Sembabule

Supportive messaging about the effectiveness of early HIV treatment was also consistently described as easing men's fears around testing for HIV and subsequent use of HIV services. This messaging was communicated by providers during pre-test counseling and sometimes during DREAMS-related programming. The message conveyed was often described as “... there is no difference between an HIV-positive person and one who is HIV-negative; they both live normally once they are given ARVs [antiretrovirals]." (Age 31, Gulu) Such messaging also was believed to facilitate timely linkage to care for those testing HIV positive.

[In the men's meeting] they told us that it's in one's best interest to know one's status so that if they are negative, they continue keeping themselves safe and if they are positive, to start medication as soon as possible. For those who had never tested for HIV, I believe they got encouraged to go and test so that they may know their status in time.
Men had a positive perception of VMMC and understood its role in HIV prevention. Many participants reported receiving VMMC several years back and were approving of DREAMS-related efforts to promote it. However, no HIV-negative men had undergone VMMC in the last two years while DREAMS was being implemented. The few who described reasons for not doing so alluded to needing more informational and emotional support to take this step.

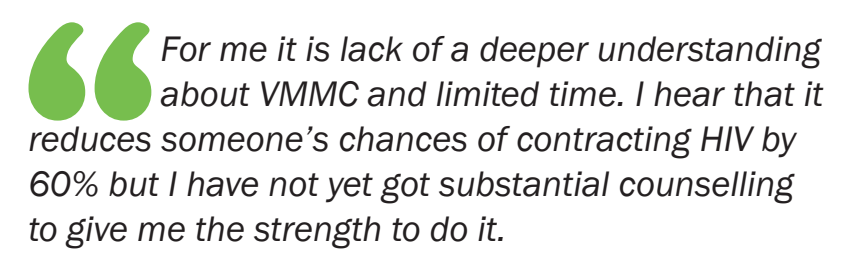

-Age 23, Gulu

\section{CARE EXPERIENCES AMONG MEN LIVING WITH HIV}

\section{Men had good engagement in HIV care and treatment.}

Nearly all of the 18 men living with HIV were currently on ART, most having started before DREAMS. Three men who had previously been in denial about an HIVpositive diagnosis years ago, which they did not trust at the time, described how DREAMS facilitated their HIV testing and subsequent direct linkage to care.

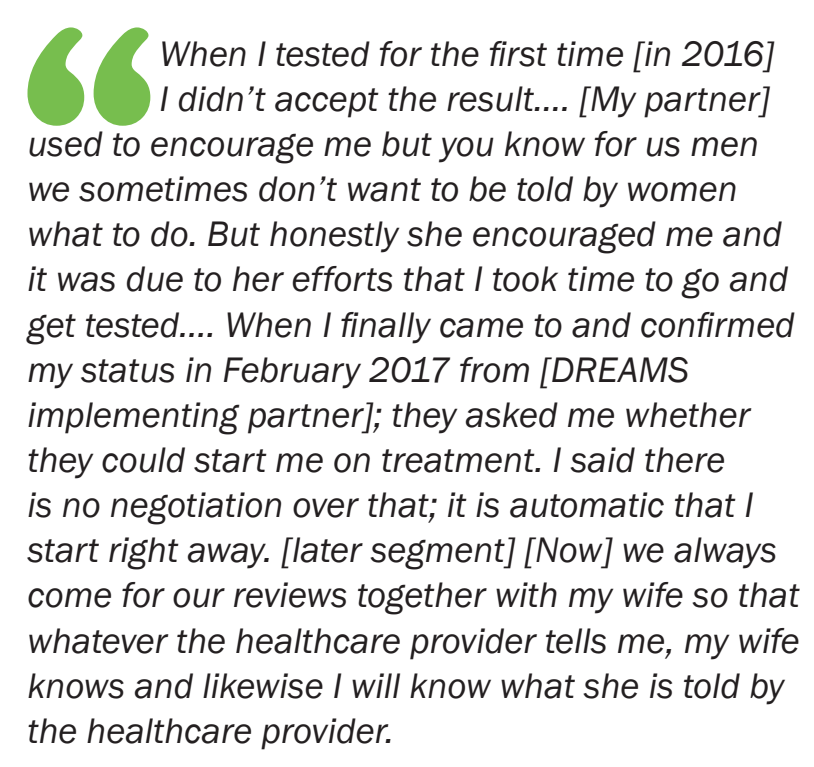

-Age 28, Gulu, living with HIV the healthcare provider. 
The first time I tested positive, I was not convinced with the result.... I said 'No' because I knew myself, I was not engaging much in sexual relationships.... It is the DREAMS Program that encouraged me to test [again for HIV]. I [also] found that my viral load had increased and they changed the kind of medicine that they were giving me. When I came to test later on, the truth is that the virus could not be detected.

-Age 28, Sembabule, living with HIV

Many men living with HIV described how their AGYW partners in DREAMS supported their retention in HIV care/treatment through emotional support as well as instrumental support, for instance by reminding them to take their treatment daily and encouraging and assisting with transportation to appointments and with retrieving ART refills. Several men noted that DREAMS had encouraged their partners to offer them such support.

She would not be with me if not for her involvement in DREAMS; that is what I think. It is not easy to be HIV negative and stay in a marriage relationship with an HIV-positive partner.

-Age 32, Gulu, living with HIV

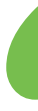

My partner...helps me to adhere to HIV treatment and care. She reminds me of my appointment dates with the health workers, also of when I am supposed to take my medicine.

-Age 46, Mukono, living with HIV

\section{Patient education was effective in increasing men's understanding of viral load/viral suppression, and some knew about treatment as prevention.}

Most men had good knowledge of viral load testing and viral suppression. About three-quarters knew their viral suppression status, among whom eight new they were virally suppressed, several reported reduced viral loads compared with previously, and one reported having a high viral load. And, when we asked, almost half knew that sustained viral suppression greatly reduces risk of transmitting HIV to their partners (i.e., TasP).
I was told that the virus in my body is no longer active, and I was told that this is a result of my being consistent with taking ARVs.

-Age 40, Mukono, living with HIV

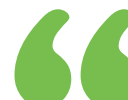

If you have been consistent in taking your medicine, when you sleep with an uninfected partner, she may not get the virus, because the medicine they give us suppresses the virus, and it sleeps so that it's not active to infect the other person.

-Age 27, Mukono, living with HIV

What you have just mentioned that taking drugs consistently reduces on the chances of infecting my partner with the virus? I have never heard this before.

-Age 44, Sembabule, living with HIV

\section{RECOMMENDATIONS}

- Overall, findings underscore the importance of meaningfully engaging men as partners, clients, and agents of change ${ }^{7}$, including within DREAMS-a program strategically aiming to reduce new HIV infections among AGYW.

\section{- Meaningful engagement as partners:}

- Address inequitable gender norms through programs that include critical reflection about gender roles and risk, last beyond a few sessions, and engage both men and women. ${ }^{8,9}$

- Directly engage men in programs alongside women (and their partners, when possible) to improve communication and mutual support, but also provide opportunities for male-only groups as safe spaces to express worries, share personal stories, and seek advice about life challenges.

\section{- Meaningful engagement as clients:}

- Continue to complement facility-based services for men with community-based, client-centered services.

- Implement supportive prevention programming that invites and meaningfully engages men (i.e., not just for purposes of ensuring they access HIV services).

- Prioritize information and messaging around HIV treatment efficacy, including TasP/ Undetectable $=$ Untransmittable $(\mathrm{U}=\mathrm{U})$, as part of promoting men's informed decision-making about their own health and preventing transmission to their partners. ${ }^{10,11}$ 


\section{- Meaningful engagement as agents of change:}

- Avoid assumptions that men are reluctant to access HIV services and prevention informationwe found that men were eager to gain information and skills to improve their relationships and reduce their risk for HIV.

- Engage men/male partners of AGYW in DREAMS as agents of change as DREAMS and other HIV prevention programming continues to be rolled out in Uganda.

\section{REFERENCES}

${ }^{1}$ Saul, J. et al. 2018. "The DREAMS core package of interventions: A comprehensive approach to preventing HIV among adolescent girls and young women," PLOS ONE 13(12): e0208167. doi: 10.1371/journal. pone.0208167

${ }^{2}$ Gottert, A. et al. 2018. "Male partners of young women in Uganda: Understanding their relationships and use of HIV testing," PLOS ONE 13(8): e0200920. doi: 10.1371/journal.pone.0200920

${ }^{3}$ Abramsky, T. et al. 2014. "Findings from the SASA! Study: a cluster randomized controlled trial to assess the impact of a community mobilization intervention to prevent violence against women and reduce HIV risk in Kampala, Uganda," BMC Medicine 12(1): 122. doi: 10.1186/ s12916-014-0122-5

${ }^{4}$ SASA! website: https://raisingvoices.org/sasa/

5ewkes, R. et al. 2008. "Impact of Stepping Stones on incidence of HIV and HSV-2 and sexual behaviour in rural South Africa: cluster randomised controlled trial," BMJ 337:a506. doi: 10.1136/bmj.a506

${ }^{6}$ Stepping Stones website: https://steppingstonesfeed back.org/

${ }^{7}$ Pulerwitz, J., A. Gottert, M. Betron, and D. Shattuck on behalf of the Male Engagement Task Force, USAID Interagency Gender Working Group (IGWG). 2019. "Do's and don'ts for engaging men \& boys.” Washington, D.C.: IGWG. 2019.

${ }^{8}$ Ruane-McAteer, E. et al. 2020. "Gender transformative programming with men and boys to improve sexual and reproductive health and rights: a systematic review of intervention studies," BMJ Global Health 5(10): e002997. doi:10.1136/bmjgh-2020-002997

${ }^{9}$ Dworkin, S. L., S. Treves-Kagan, and S. A. Lippman. 2013. “Gendertransformative interventions to reduce HIV risks and violence with heterosexually-active men: a review of the global evidence," AIDS and Behavior 17(9): 2845-63. doi: 10.1007/s10461-013-0565-2

${ }^{10}$ Lippman, S. A. et al. 2020. "Treatment as prevention-provider knowledge and counseling lag behind global campaigns," Journal of Acquired Immune Deficiency Syndromes 83(2): e9-e12. doi: 10.1097/ QAI.0000000000002197

${ }^{11}$ Mooney, A. C. et al. 2017. “Men's perceptions of treatment as prevention in South Africa: implications for engagement in HIV care and treatment," AIDS Education and Prevention 29(3): 274-87. doi: 10.1521/aeap.2017.29.3.274
For more information about the DREAMS IS portfolio:

Visit the DREAMS IS special series page: https:// knowledgecommons.popcouncil.org/series_dreams/

"How to reduce HIV risk among adolescent girls and young women in sub-Saharan Africa? Implementation science around the DREAMS Initiative," DREAMS Project Brief. doi: 10.31899/hiv7.1009

"Reducing HIV risk among young women and their partners: highlights from the DREAMS implementation science research portfolio," DREAMS Project Brief. doi: 10.31899/hiv11.1026

For more information about the study in Uganda:

"Effective engagement of male partners of adolescent girls and young women in HIV services: implementation science research in Uganda," DREAMS Activity Brief. doi: 10.31899/hiv6.1012

"Understanding the relationships and use of HIV testing among male partners of adolescent girls and young women in Uganda: Findings from implementation science research," DREAMS Uganda Results Brief.

“'A man without money getting a sexual partner? It doesn't exist in our community': male partners' perspectives on transactional sexual relationships in Uganda and Eswatini," Culture, Health \& Sexuality 1-16. doi: 10.1080/13691058.2021.1904521

"Male partners of young women in Uganda: Understanding their relationships and use of HIV testing," PLOS ONE 13(8): e0200920. doi: 10.1371/ journal.pone.0200920

\section{Study contacts:}

Ann Gottert (agottert@popcouncil.org) and Julie Pulerwitz (jpulerwitz@popcouncil.org)
POPULATION COUNCIL

Ideas. Evidence. Impact.
The Population Council confronts critical health and development issues-from stopping the spread of HIV to improving reproductive health and ensuring that young people lead full and productive lives. Through biomedical, social science and public health research in about 50 countries, the Council works with our partners to deliver solutions that lead to more effective policies, programs, and technologies to improve lives worldwide. Established in 1952 and headquartered in New York, the Council is a nongovernmental, nonprofit organization with an international board of trustees. popcouncil.org

Suggested citation: Population Council. 2021. "Hearing from men in Uganda: experiences with HIV services and prevention programming, and perceptions of DREAMS-findings from DREAMS implementation science research," Uganda DREAMS Results Brief. Washington, DC: Population Council. 